\title{
Liberal Manipulation of Ventilator Settings and Its Impact on Tracheostomy Rate and Ventilator-Free Days
}

\author{
Ariel M Modrykamien MD, Lori Killian RRT-NPS, and Ryan W Walters MSc
}

\begin{abstract}
BACKGROUND: The utilization of checklists, bundles, and protocols attempts to provide standardization in the delivery of patient care. Despite important progress obtained in the prevention of hospitalacquired infections, the daily management of mechanical ventilation is still prone to heterogeneity, depending on the number of providers manipulating the ventilator. Whether the number of changes made on ventilator parameters impacts clinical outcomes remains unknown. METHODS: A quality improvement project was designed to assess whether liberal manipulations of ventilator settings affect the rate of tracheostomy and 28 ventilator-free days. Over the course of $7 \mathrm{~d}$, respiratory therapists recorded all ventilator changes in newly ventilated subjects. Ventilator changes were considered as major changes if manipulations included changes in the mode of ventilation. Minor changes included manipulations of settings within the same mode of ventilation. We evaluated whether the number of total and major changes affected clinical outcomes. Logistic regression was used for multivariate analysis. RESULTS: One-hundred seventeen ventilator manipulations were recorded among 54 subjects. Of those 117 ventilator changes, $35 \%$ were major manipulations. For every major ventilator manipulation, the odds of requiring tracheostomy increased 4.95 times. Furthermore, for every major ventilator change, there was an $18.6 \%$ decrease in 28 ventilator-free days. These associations were found after adjustments by APACHE (Acute Physiology and Chronic Health Evaluation) II score, body mass index, and type of ICU. The total number of changes was not associated with either primary outcome measure. CONCLUSIONS: The number of major ventilator manipulations is associated with rate of tracheostomy and stay on the ventilator. Key words: mechanical ventilation; protocols; outcomes; tracheostomy. [Respir Care 2016;61(1):30-35. () 2016 Daedalus Enterprises]
\end{abstract}

\section{Introduction}

Homogeneity in the way health-care providers deliver patient care has been associated with improvements in clinical outcomes. In fact, the application of checklists to standardize processes of care has become common prac-

\footnotetext{
Dr Modrykamien and Ms Killian are affiliated with the Respiratory Care Department, Baylor University Medical Center, Dallas, Texas. Mr Walters is affiliated with the Division of Clinical Research and Evaluative Science, Creighton University School of Medicine, Omaha, Nebraska.

The authors have disclosed no conflicts of interest.

Correspondence: Ariel M Modrykamien MD, Medical Director, Respiratory Care Services, Pulmonary and Critical Care Division, Baylor University Medical Center, 3600 Gaston Ave. Wadley Tower, Suite 960, Dallas, TX 75246. E-mail: ariel.modrykamien@baylorhealth.edu.
}

DOI: $10.4187 /$ respcare. 03887 tice in multiple disciplines. For example, central line and ventilator bundles and ICU rounding and transportation checklists are usual tools meant to enhance patient safety and institutional quality. ${ }^{1-3}$ Despite important progress obtained in terms of standardization of medical practice, based on evidence-based medicine guidelines, bundles, and checklists, gaps still remain in many areas. Mechanical ventilation, which includes a vast and complicated terminology and a variety of ventilator modes and setting options, is perfectly set to generate heterogeneity in the process of ventilating patients. The recognition of this important limitation brought about new technology, such as modes of closed-loop ventilation, which allow ventilators to automatically adjust parameters to achieve certain rules (ie, automatic modification of driving pressure to achieve a desired tidal volume).4-6 Nevertheless, although the aforementioned technology may reduce variability, it is available only in a limited number of ventilators, and its use remains provider-dependent. 
Variability in the process of ventilating patients may also be influenced by the type of ICU model. Intensive care units staffed with an open model, allowing multiple consultants to initiate or modify ventilator orders, might also increase heterogeneity and, eventually, affect clinical outcomes. In order to assess the association between the number of ventilator changes in an open ICU, a strategy that we have called liberal manipulation of ventilator settings, and its impact on certain clinical outcomes, we undertook the present quality improvement project.

\section{Methods}

\section{Procedures}

Ventilator management in our organization is based on a liberal strategy, which implies that health-care providers of different specialties are allowed to change ventilator settings according to their clinical assessments and interpretations. Specifically, a variety of physicians may change settings and/or operate ventilators themselves, or they may do it through orders executed by a respiratory therapist and/or nurses. Consequently, mechanically ventilated patients may be exposed to a variety of ventilator modes and parameters in a relatively short period of time. Based on the perception within the Respiratory Care Department that a liberal manipulation of ventilator settings may have been affecting clinical outcomes, we designed a quality improvement project to assess the aforementioned hypothesis. Our project involves 3 phases: (1) collection of data during a period of liberal strategy; (2) writing and implementation of a mechanical ventilation changes policy; and (3) collection of data during a period of restrictive strategy (post-policy implementation). In this article, we present the results obtained during the initial phase of the quality improvement project, which included data collected during the liberal strategy period. This project has been exempted from institutional review board evaluation.

Over 7 consecutive days during the month of September 2014, respiratory therapists recorded all ventilator changes made on all newly mechanically ventilated subjects admitted in any of the 120 ICU beds at Baylor University Medical Center. Only $7 \mathrm{~d}$ were assessed, as the daily rate of newly mechanically ventilated patients in our institution is between 10 and 15 patients. Therefore, a priori, we assumed that we would include approximately 100 subjects over that period of time. Once ventilator changes were collected, they were subsequently divided into major changes, if ventilator manipulations involved changes in the mode of ventilation (ie, volume control to pressure control), and minor changes, if manipulations consisted of changes in parameters within the same mode of ventilation (ie, increases in $\mathrm{F}_{\mathrm{IO}_{2}}$ ). Importantly, changes from continuous mandatory ventilation/volume control+ (pressure-

\section{QUICK LOOK}

\section{Current knowledge}

Weaning from mechanical ventilation has undergone whole-scale changes in the last decade and is more commonly known as liberation from the ventilator. Daily spontaneous breathing trials coupled with spontaneous awakening trials represent the standard of care. Frequent ventilator manipulations in a deliberate weaning process have not been shown to speed discontinuation from mechanical ventilation.

\section{What this paper contributes to our knowledge}

In a prospective observational trial of 54 subjects, more frequent ventilator manipulations by the multidisciplinary staff were associated with more frequent use of tracheostomy and longer duration of mechanical ventilation. In this quality improvement project, it is not known whether this was causal or if the more frequent ventilator manipulations were related to severity of respiratory dysfunction.

regulated volume control ventilation) to continuous mandatory ventilation/volume control or from synchronized intermittent mandatory ventilation/volume control to continuous mandatory ventilation/volume control and vice versa were considered major changes, despite the fact that target or limited tidal volumes remained the same. All subjects were ventilated with a Puritan Bennett 840 ventilator (Covidien, Minneapolis, Minnesota).

Ventilator changes associated with patient transportation (eg, to computed tomography scan) or those made for the purpose of ventilator weaning (eg, continuous mandatory ventilation mode to pressure support ventilation of $5 \mathrm{~cm} \mathrm{H}_{2} \mathrm{O}$ ) were not included in the analysis. The reasons why providers decided to change ventilator settings were not recorded. Subjects' demographics, type of ICU in which subjects were admitted, and reason for admission were recorded as well. The outcomes of interest were rate of tracheostomy during present hospitalization and 28 ventilator-free days. This last outcome was calculated by subtracting total days on mechanical ventilation from $28 \mathrm{~d}$. Subjects who expired during the hospitalization or were discharged to long-term acute care facilities on mechanical ventilation were assigned zero ventilator-free days.

\section{Statistical Analysis}

Continuous demographic and clinical variables are presented as mean $\pm \mathrm{SD}$, whereas categorical variables are presented as frequency and percent. Ventilator-free days 
was considered a count variable. A negative binomial model fit significantly better than a Poisson model $(-2 \Delta \mathrm{LL}=410.3, P<.05$; scale $=1.9)$, but because approximately $29.6 \%$ of subjects had zero ventilator-free days, a zero-inflated negative binomial model was preferred $(-2 \Delta \mathrm{LL}=49.3, P<.05)$. Logistic regression was used for tracheostomy. Both models evaluated whether the number of ventilator changes or number of major ventilator changes predicted the outcome after adjusting for body mass index, APACHE (Acute Physiology and Chronic Health Evaluation) II score, and type of ICU (ie, medical, surgical, cardiac, or neurological). For analysis, continuous covariates were centered near their means to allow meaningful interpretations of the intercept. All analyses were conducted using SAS 9.3 (SAS Institute, Cary, North Carolina); 2-tailed $P<.05$ was considered statistically significant.

\section{Results}

Over the course of the study period, 117 ventilator manipulations were recorded in 54 subjects. Twenty-nine $(53.7 \%)$ subjects were men, and the average age was $53 \pm$ $14 \mathrm{y}$. Of the total ventilator manipulations, $41(35 \%)$ were major ventilator changes. Twenty-nine subjects (54\%) had major ventilator changes, ranging from 1 to 3 major ventilator changes/subject. Only $9(20 \%)$ subjects had $>1$ major ventilator change (Table 1). These changes were mostly seen over the first $48 \mathrm{~h}$ after intubation. Demographic and clinical variables are presented in Table 2, whereas the most common reasons for ICU admission are presented in Table 3. As shown, some diagnoses were grouped together for simplicity (ie, angina, heart failure, and coronary artery bypass graft). Descriptions of major ventilator changes and the number and percentage of subjects who received each one of them are shown in Table 4. Table 1 shows clinical outcomes of subjects $(n=29)$ who had at least one major ventilator change made. Final model results are presented in Tables 5 and 6 .

For the outcome 28 ventilator free days, the number of major ventilator changes significantly predicted the number of ventilator-free days, with every additional major ventilator change resulting in an $18.6 \%$ decrease in ventilator-free days after adjusting for the demographic and clinical variables $(95 \% \mathrm{CI}=7.1-28.6 \%)$.

For tracheostomy, every additional major ventilator change resulted in a 4.9 times increase in the odds of requiring a tracheostomy $(95 \% \mathrm{CI}=1.6-15.1)$. None of the outcomes of interest was associated with total ventilator changes, even after adjusting for other variables.

\section{Discussion}

The evaluation of the first phase of our quality improvement project shows the following results. (1) The number
Table 1. Number of Major Ventilator Changes per Subject, 28 Ventilator-Free Days, Need for Tracheostomy, and Death $(n=29)$

\begin{tabular}{|c|c|c|c|c|}
\hline $\begin{array}{l}\text { Subject } \\
\text { no. }\end{array}$ & $\begin{array}{c}\text { Major Changes } \\
\text { per Subject }\end{array}$ & $\begin{array}{l}28 \text { Ventilator- } \\
\text { Free Days }\end{array}$ & Tracheostomy & $\begin{array}{l}\text { Hospital } \\
\text { Death }\end{array}$ \\
\hline 1 & 1 & 15 & No & No \\
\hline 2 & 1 & 1 & Yes & No \\
\hline 3 & 1 & 20 & Yes & No \\
\hline 4 & 1 & 26 & No & No \\
\hline 5 & 1 & 0 & Yes & No \\
\hline 6 & 1 & 26 & No & No \\
\hline 7 & 1 & 14 & No & No \\
\hline 8 & 1 & 19 & Yes & No \\
\hline 9 & 1 & 24 & No & No \\
\hline 10 & 1 & 20 & No & No \\
\hline 11 & 1 & 23 & No & No \\
\hline 12 & 1 & 19 & No & No \\
\hline 13 & 1 & 19 & Yes & No \\
\hline 14 & 1 & 22 & No & No \\
\hline 15 & 1 & 0 & Yes & Yes \\
\hline 16 & 1 & 26 & No & No \\
\hline 17 & 1 & 21 & Yes & No \\
\hline 18 & 1 & 0 & Yes & No \\
\hline 19 & 1 & 24 & No & No \\
\hline 20 & 1 & 3 & Yes & No \\
\hline 21 & 2 & 17 & Yes & No \\
\hline 22 & 2 & 0 & Yes & Yes \\
\hline 23 & 2 & 0 & Yes & Yes \\
\hline 24 & 2 & 0 & Yes & Yes \\
\hline 25 & 2 & 0 & No & Yes \\
\hline 26 & 2 & 0 & Yes & No \\
\hline 27 & 3 & 8 & Yes & No \\
\hline 28 & 3 & 4 & Yes & No \\
\hline 29 & 3 & 7 & Yes & No \\
\hline
\end{tabular}

of major ventilator changes performed during initial days of mechanical ventilation is directly associated with the rate of tracheostomy. (2) The number of major ventilator changes is inversely associated with 28 ventilator-free days. In other words, the higher the number of changes in the mode of mechanical ventilation, the longer subjects remain on mechanical ventilation.

This study represents the first description of the implications of liberal manipulations of ventilators in clinical outcomes. It also adds to the current literature, as there are no prior reports on this relevant topic. Furthermore, it provides a better understanding of the consequences of having certain staffing models and practices in individual ICUs. Whereas liberal manipulation of ventilator settings may provide flexibility in modifying parameters according to patients' changes, it also increases heterogeneity in ventilator practices with eventual undesirable outcomes. Hence, in times in which outcome-driven rewards (and punishment) have become the norm in medicine practice, 
Table 2. Demographic and Clinical Variables and Outcomes

\begin{tabular}{|c|c|c|c|c|}
\hline & $\begin{array}{l}\text { All Subjects } \\
(\mathrm{N}=54)\end{array}$ & $\begin{array}{l}\text { Major Changes } \\
\quad(n=29)\end{array}$ & $\begin{array}{c}\text { No Major Changes } \\
(n=25)\end{array}$ & $P^{*}$ \\
\hline Body Mass Index, $\mathrm{kg} / \mathrm{m}^{2}$ & $30.8 \pm 8.2$ & $31.6 \pm 7.6$ & $29.9 \pm 9.0$ & .46 \\
\hline APACHE II & $21.4 \pm 5.3$ & $21.3 \pm 5.6$ & $21.5 \pm 5.1$ & .87 \\
\hline No. of ventilator changes $\dagger$ & $2.2 \pm 1.7$ & $2.7 \pm 2.0$ & $1.5 \pm 0.8$ & .01 \\
\hline No. of major ventilator changes $\ddagger$ & $0.7 \pm 0.8$ & $1.4 \pm 0.7$ & & \\
\hline 28 ventilator-free days & $13.0 \pm 10.3$ & $12.3 \pm 10.2$ & $13.9 \pm 10.4$ & .59 \\
\hline Tracheostomy & $23(43)$ & $17(59)$ & $6(24)$ & .01 \\
\hline Mortality & $9(17)$ & $5(17)$ & $4(16)$ & $>.99$ \\
\hline \multicolumn{5}{|l|}{ Type of ICU } \\
\hline Medical & $25(46)$ & $11(38)$ & $14(56)$ & .27 \\
\hline Surgical & $16(30)$ & $10(34)$ & $6(24)$ & .55 \\
\hline Cardiac & $5(9)$ & $3(10)$ & $2(8)$ & $>.99$ \\
\hline Neurological & $8(19)$ & $5(17)$ & $3(12)$ & .71 \\
\hline \multicolumn{5}{|c|}{$\begin{array}{l}\text { Values shown are mean } \pm \mathrm{SD} \text { or } n(\%) \text {. } \\
* P \text { values are for comparison between subjects with and without major changes. } \\
\dagger \text { Range }=1-10 \text {. } \\
\text { \#Range }=0-3 \text {. } \\
\text { APACHE II = Acute Physiology and Chronic Health Evaluation II }\end{array}$} \\
\hline
\end{tabular}

Table 3. Reasons for ICU Admission

\begin{tabular}{lc}
\hline \hline \multicolumn{1}{c}{ Diagnoses } & $n(\%)$ \\
\hline Mental status change/stroke/ICH & $13(24 \%)$ \\
Respiratory failure & $11(20 \%)$ \\
Abdominal surgery & $6(11 \%)$ \\
Sepsis/infections & $5(9 \%)$ \\
Heart failure/angina/coronary artery bypass graft & $5(9 \%)$ \\
Trauma & $5(9 \%)$ \\
Neck surgery & $5(9 \%)$ \\
Gastrointestinal bleeding & $2(4 \%)$ \\
Cardiac arrest & $1(2 \%)$ \\
Solid organ transplant & $1(2 \%)$
\end{tabular}

$\overline{\mathrm{ICH}}=$ intracerebral hemorrhage

standardization of processes based on evidence-based medicine should prevail.

In the critical care literature, there is abundant evidence supporting the use of a variety of bundles, checklists, and protocols. ${ }^{7}$ Specifically, the Keystone ICU project, which focused on interventions to reduce the incidence of catheter-related bloodstream infections, utilized evidence-based checklists recommended by the Centers for Disease Control and Prevention (ie, handwashing, use of full-barrier precautions, etc.) plus a daily goal sheet for improvement of communication. One-hundred three ICUs participated, adopting the aforementioned standardized measures. As a result, the rate of catheter-related bloodstream infections decreased from 7.7/1,000 catheter days at baseline to 1.4/1,000 catheter days 18 months after the implementation of the previously described interventions. ${ }^{1}$
Table 4. Description of Major Ventilator Changes and Number of Subjects Exposed to Them

\begin{tabular}{cccc}
\hline \hline $\begin{array}{c}\text { Initial Mechanical } \\
\text { Ventilation Mode }\end{array}$ & $\begin{array}{c}\text { Final Mechanical } \\
\text { Ventilation Mode }\end{array}$ & $\begin{array}{c}\text { Changes, } \\
n(\%)\end{array}$ & $\begin{array}{c}\text { Subjects, } \\
n(\%)\end{array}$ \\
\hline CMV/VC & PSV & $12(29)$ & $7(24)$ \\
Bilevel & PSV & $5(13)$ & $3(11)$ \\
PSV & CMV/VC & $5(13)$ & $3(11)$ \\
PSV & Bilevel & $4(10)$ & $2(7)$ \\
SIMV/VC & PSV & $3(7)$ & $2(7)$ \\
CMV/PC & SIMV/VC & $2(5)$ & $2(7)$ \\
Bilevel & CMV/VC & $2(5)$ & $2(7)$ \\
CMV/PC & PSV & $2(5)$ & $2(7)$ \\
VC & CMV/PC & $2(5)$ & $2(7)$ \\
PSV & CMV/PC & $1(2)$ & $1(3)$ \\
SIMV/VC & CMV/PC & $1(2)$ & $1(3)$ \\
CMV/VC & SIMV/VC & $1(2)$ & $1(3)$ \\
CMV/VC & Bilevel & $1(2)$ & $1(3)$ \\
& & & \\
CMV $=$ continuous mandatory ventilation & & \\
VC $=$ volume control \\
$\begin{array}{l}\text { PSV }=\text { pressure support ventilation } \\
\text { SIMV }=\text { synchronized intermittent mandatory ventilation } \\
\text { PC }=\text { pressure control }\end{array}$ \\
\hline
\end{tabular}

In the area of mechanical ventilation, the application of a ventilator bundle, composed by head elevation, oral decontamination with chlorhexidine, deep venous thrombosis prophylaxis, and peptic ulcer disease prophylaxis also showed reductions in the rate of ventilator-associated pneumonia. ${ }^{8,9}$ Furthermore, liberation from mechanical ventilation utilizing a stepwise strategy, which includes (1) awakening and breathing coordination with the utilization of a weaning protocol, (2) delirium monitoring and manage- 
Table 5. Ventilator-Free Days: Zero-Inflated Negative Binomial Regression Results $(n=54)$

\begin{tabular}{|c|c|c|c|c|}
\hline & $\begin{array}{l}\text { Regression } \\
\text { Coefficient }\end{array}$ & SE & Ratio & $P$ \\
\hline Intercept & 2.97 & 1.14 & & \\
\hline APACHE II $(0=20)$ & 0.00 & 0.01 & 1.01 & .77 \\
\hline Body mass index $(0=30)$ & -0.03 & 0.01 & 0.98 & .001 \\
\hline \multicolumn{5}{|l|}{ Type of ICU* } \\
\hline Medical & 0.01 & 0.15 & 1.01 & .97 \\
\hline Surgical & 0.09 & 0.16 & 1.10 & .57 \\
\hline Cardiac & 0.27 & 0.22 & 1.30 & .22 \\
\hline Major ventilator changes & -0.21 & 0.07 & 0.81 & .002 \\
\hline \multicolumn{5}{|c|}{$\begin{array}{l}\text { Zero-model intercept }=-0.8651, \mathrm{SE}=0.30 \text {, scale }=0.033 \text {. } \\
\text { * Regression coefficients are relative to neurological ICU. Taking as baseline neurological } \\
\text { ICU, all other coefficients were calculated. } \\
\text { APACHE II = Acute Physiology and Chronic Health Evaluation II }\end{array}$} \\
\hline
\end{tabular}

Table 6. Tracheostomy: Logistic Regression Results $(n=54)$

\begin{tabular}{lcccc}
\hline \hline & $\begin{array}{c}\text { Regression } \\
\text { Coefficient }\end{array}$ & SE & $\begin{array}{r}\text { Odds } \\
\text { Ratio }\end{array}$ & $P$ \\
\hline Intercept & -3.94 & 1.49 & & \\
APACHE II $(0=20)$ & -0.05 & 0.07 & 0.95 & .45 \\
Body mass index $(0=30)$ & 0.12 & 0.05 & 1.13 & .02 \\
Type of ICU* & & & & \\
$\quad$ Medical & 2.70 & 1.45 & 14.82 & .063 \\
$\quad$ Surgical & 3.07 & 1.50 & 21.58 & .04 \\
$\quad$ Cardiac & 1.68 & 1.71 & 5.39 & .33 \\
Major ventilator changes & 1.60 & 0.57 & 4.95 & .02
\end{tabular}

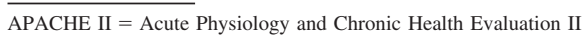

* Regression coefficients are relative to neurological ICU. Taking as baseline the neurological ICU, all other coefficients were calculated.

ment, and (3) early exercise and mobility, demonstrated reductions in time spent on the ventilator and incidence of delirium. ${ }^{10-15}$ The use of this bundle also showed a higher likelihood of discharge home (rather than to nursing facilities) and survival. ${ }^{12}$ Therefore, based on the aforementioned data, it seems prudent and effective to adopt standardized systems to obtain desirable outcomes.

Our study shows that the liberal manipulation of ventilator settings by multiple providers is associated with heterogeneity in practice and major ventilator changes in short periods of time. Major ventilator manipulations were associated with a higher rate of tracheostomy and increased stay on the ventilator. Despite these important associations, several limitations of the study preclude further conclusions. First, the number of subjects included in the study was relatively small due to the limited time in which data collection was performed. During the planning phase of the quality improvement project, under the assumption that 15 newly mechanically ventilated patients would be admitted on a daily basis to our ICUs, we aimed to include
100-105 subjects. Unfortunately, only 54 subjects were able to be included. Therefore, a larger number of subjects, with other demographics and/or levels of severity, could have modified our results. Second, the retrospective nature of our analysis may have exposed this study to selection or information bias. It is possible that some patients may have not been included due to a variety of reasons, such as patient transportation during data collection, transfers between units, etc. Also, it is possible that some parameters or modes of mechanical ventilation escaped data collection due to lack of registration. Third, it is likely that the need for major ventilator changes may have been a manifestation of poor patient-ventilator synchrony. Hence, the rate of tracheostomy may have been associated with this asynchrony rather than with ventilator changes. Fourth, the statement that major ventilator changes are associated with worse clinical outcomes may be an oversimplification. On many occasions, as patients' respiratory physiology changes, mechanical ventilator settings should be modified as well. In those situations, it is unlikely that major ventilator changes would be associated with worse outcomes. In our study, we assumed that major ventilator changes were a consequence of a model of liberal ventilator manipulation rather than patient physiologic changes. This assumption could have been incorrect. Last, we have not collected the actual reasons for major ventilator changes. Therefore, the premise that major ventilator changes were linked to liberal manipulation of ventilators was an assumption that remains to be confirmed.

\section{Conclusions}

The first phase of this quality improvement project showed that the number of major changes is directly associated with rate of tracheostomy and stay on mechanical ventilation. The implementation of a ventilator management policy and further evaluation before and after its implementation will provide important information regarding this topic.

\section{REFERENCES}

1. Pronovost P, Needham D, Berenholtz S, Sinopoli D, Chu H, Cosgrove $\mathrm{S}$, et al. An intervention to decrease catheter-related bloodstream infections in the ICU. N Engl J Med 2006;355(26):27252732 .

2. Weiss CH, Moazed F, McEvoy CA, Singer BD, Szleifer I, Amaral LA, et al. Prompting physicians to address a daily checklist and process of care and clinical outcomes: a single-site study. Am J Respir Crit Care Med 2011;184(6):680-686.

3. Simpson SQ, Peterson DA, O'Brien-Ladner AR. Development and implementation of an ICU quality improvement checklist. AACN Adv Crit Care 2007;18(2):183-189.

4. Lellouche F, Mancebo J, Jolliet P, Roeseler J, Schortgen F, Dojat M, et al. A multicenter randomized trial of computer-driven protocolized weaning from mechanical ventilation. Am J Respir Crit Care Med 2006;174(8):894-900. 


\section{Liberal Manipulation of Ventilator Settings}

5. Branson R. Understanding and implementing advances in ventilator capabilities. Curr Opin Crit Care 2004;10(1):23-32.

6. Burns KE, Lellouche F, Lessard MR. Automating the weaning process with advanced closed-loop systems. Intensive Care Med 2008; 34(10):1757-1765.

7. Marwick C, Davey P. Care bundles: the holy grail of infectious risk management in hospital? Curr Opin Infect Dis 2009;22(4):364-369.

8. Wip C, Napolitano L. Bundles to prevent ventilator-associated pneumonia: how valuable are they? Curr Opin Infect Dis 2009;22(2):159166.

9. Rello J, Afonso E, Lisboa T, Ricart M, Balsera B, Rovira A, et al. A care bundle approach for prevention of ventilator-associated pneumonia. Clin Microbiol Infect 2013;19(4):363-369.

10. Morandi A, Brummel NE, Ely EW. Sedation, delirium and mechanical ventilation: the "ABCDE" approach. Curr Opin Crit Care 2011; 17(1):43-49.

11. Girard TD, Kress JP, Fuchs BD, Thomason JW, Schweickert WD, Pun BT, et al. Efficacy and safety of a paired sedation and ventilator weaning protocol for mechanically ventilated patients in intensive care (Awakening and Breathing Controlled trial): a randomised controlled trial. Lancet 2008;371(9607):126-134.

12. Schweickert WD, Pohlman MC, Pohlman AS, Nigos C, Pawlik AJ, Esbrook CL, et al. Early physical and occupational therapy in mechanically ventilated, critically ill patients: a randomised controlled trial. Lancet 2009;373(9678):1874-1882.

13. Balas MC, Burke WJ, Gannon D, Cohen MZ, Colburn L, Bevil C, et al. Implementing the awakening and breathing coordination, delirium monitoring/management, and early exercise/mobility bundle into everyday care: opportunities, challenges, and lessons learned for implementing the ICU Pain, Agitation, and Delirium Guidelines. Crit Care Med 2013;41(9):S116-S127.

14. Klompas M, Anderson D, Trick W, Babcock H, Kerlin MP, Li L, et al. The preventability of ventilator-associated events: the CDC Prevention Epicenters Wake Up and Breathe Collaborative. Am J Respir Crit Care Med 2015;191(3):292-301.

15. Macintyre NR. Evidence-based assessments in the ventilator discontinuation process. Respir Care 2012;57(10):1611-1618.

This article is approved for Continuing Respiratory Care Education credit. For information and to obtain your CRCE

(free to AARC members) visit

www.rcjournal.com 